\title{
ESTUDIO DEL HORIZONTE LOCAL
}

Resumen: El estudio del horizonte es fundamental para poder facilitar las primeras observaciones de los alumnos en un centro educativo. Un simple modelo, que debe realizarse para cada centro, nos permite facilitar el estudio y la comprensión de los primeros rudimentos astronómicos. El modelo construido se presenta a su vez como un sencillo modelo de reloj ecuatorial y a partir de él se pueden construir otros modelos (horizontal y vertical).

Palabras clave: Horizonte. Reloj ecuatorial.

\section{ESTUDO DO HORIZONTE LOCAL}

Resumo: O estudo do horizonte é fundamental para facilitar as primeiras observações dos alunos num centro educativo. Um modelo simples, que deve ser feito para cada centro, permite facilitar o estudo e a compreensão dos primeiros rudimentos astronômicos. O modelo construído apresenta-se, por sua vez, como um modelo simples de relógio equatorial e a partir dele pode-se construir outros modelos (horizontal e vertical)

Palavras-chave: Horizonte. Relógio equatorial.

\section{STUDY OF THE LOCAL HORIZON}

\begin{abstract}
The study of the horizon is fundamental to easy the first observations of the students at any education center. A simple model, to be developed in each center, allows to easy the study and comprehension of the rudiments of astronomy. The constructed model is presented in turn as a simple equatorial clock, other models (horizontal and vertical) may be constructed starting from it.
\end{abstract}

Keywords: Horizon. Equatorial clock.

\section{Introduccíon}

El pasado mes de junio de 2009 la IAU, Unión Astronómica Internacional, en colaboración con la UNESCO organizaron un par de cursos de formación del profesorado en Ecuador y Perú. Esta es una experiencia piloto dentro del marco del nuevo programa NASE, Network for Astronomy Schools Education, que empezara a desarrollarse a partir del 2010 en todos los países que deseen sumarse a este proyecto de la IAU. El principal objetivo del mismo consiste en formar en astronomía a los docentes de primara y secundaria que lo deseen. Los cursos se imparten en la lengua del país. Sus contenidos responden a una formación básica en astronomía para los enseñantes y la metodología consiste básicamente en un conjunto de talleres con actividades prácticas para desarrollar durante los mismos. En la sección siguiente se presentara a modo de ejemplo uno de estos talleres. Antes, pero, se relatara un breve resumen y valoración de los cursos realizados.

Aunque el proyecto prepara cursos cuya duración será de una semana, estos cursos pilotos solo duraron 3 días. En ellos se desarrollaron 3 conferencias, ocho talleres, sesiones de discusión y evaluación y las observaciones que el tiempo permitió llevar a cabo.

\footnotetext{
${ }^{1}$ Universidad Politécnica de Cataluña (Barcelona, España)
} 
El curso en Ecuador tuvo lugar en Salinas de 18 al 20 de junio en colaboración con el Observatorio Astronómico de Quito. Del 25 al 27 de junio se desarrollo en Lima, en la Universidad de San Marcos. En ambos casos estos cursos eran la primera experiencia para más del $80 \%$ de los asistentes. Su valoración fue muy satisfactoria y la metodología empleado muy exitosa. Todos los asistentes recibieron una publicación con todos los talleres completos y un resumen de las conferencias. También se ofrecía material complementario en las páginas web <http:/www.csic.es/unawe $>$ y $<$ http://www.csic.es/astrosecundaria $>$ de primaria y secundaria respectivamente.

\section{La Observacíon}

Los profesores de muchas ramas de la ciencia (física, química, biología, etc.) pueden decir que no es posible trabajar de forma correcta en un centro de secundaria si no se dispone de un laboratorio. En este sentido, procede que los profesores de astronomía estén contentos porque ellos siempre disponen de un "laboratorio de astronomía". Todos los institutos y escuelas tiene un sitio para los juegos de sus alumnos: el patio. El patio no es solo un punto de recreo, es también un laboratorio de astronomía: es un lugar que ofrece la posibilidad de llevar a cabo en él actividades prácticas de astronomía. Puesto que si en toda escuela o instituto se dispone de un laboratorio, iparece oportuno usarlo!

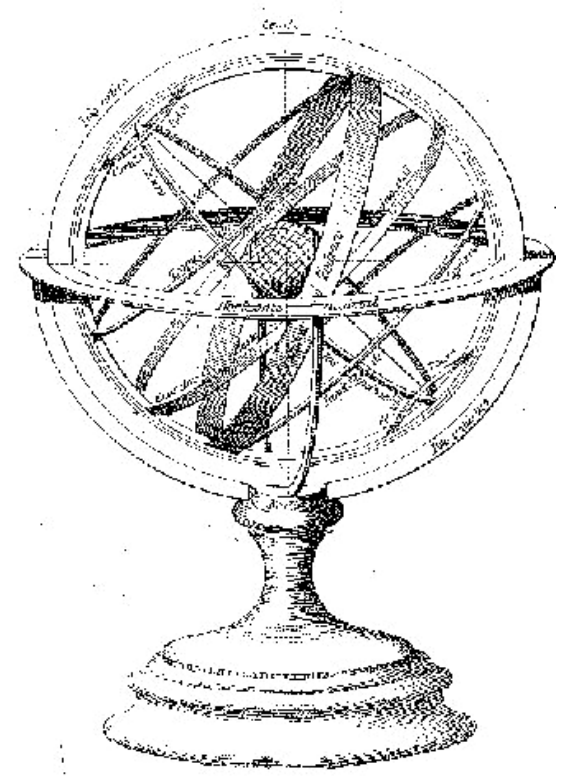

Figura 1: Representación clásica de la esfera celeste.

Si se introducen los primeros conceptos de astronomía sobre la esfera celeste siguiendo un esquema clásico pueden surgir algunos problemas al realizar las primeras observaciones. La esfera celeste, pensada como hacían los antiguos griegos, como una esfera "transparente" donde están situadas todos los astros sigue siendo utilizada en nuestros días. Ya sabemos que es solo una representación útil, y que no existe realmente, pero resulta cómodo pensar en ella. Se define pues, como una esfera de radio infinito cuyo centro es la Tierra. Los elementos que definimos sobre nuestro planeta: 
ecuador, meridianos y paralelos se extiende de forma natural como prolongación de los planos de intersección con la Tierra o con la esfera celeste.

Un problema que surge cuando el estudiante se acerca al patio para desarrollar desde él actividades prácticas de astronomía, es la diferente situación relativa de la esfera celeste (Figura 1) cuando el profesor explica dentro del recinto de una clase y cuando explica fuera de ella, en el patio del colegio.

Cuando el profesor, sobre la pizarra, textos o maquetas, habla de meridianos y paralelos, de coordenadas de posición, presenta figuras parecidas a la Figura 1. Lo anterior, no representa mayor dificultad y los estudiantes pueden entender sin problemas. Las figuras que tienen los alumnos ante sus ojos son análogas a las que han usado cuando estudiaban geografía (Figura 2).

Los problemas comienzan cuando estamos observando y no aparece ninguna línea en el cielo. No se puede ver el eje de rotación y no es sencillo encontrar referencias en el firmamento. El principal escollo es que ahora el estudiante está situado en el interior de la esfera celeste mientras que hemos presentado toda la información en la clase mirando el cielo desde el exterior de la esfera celeste. Entonces no resulta fácil comprender la nueva situación vista desde dentro (Figura 3).

Obviamente después de esta experiencia podríamos pensar en un cambio de nuestra presentación en el aula. Es posible hacer en el aula de clase una presentación desde el punto de vista del interior de la esfera. Esta forma de ver las cosas es más similar a la situación real del observador, pero no es bueno sólo ofrecer esta presentación. Los estudiantes deben ser capaces de poder leer cualquier libro de astronomía y poder entender la abstracción correspondiente a la observación de la esfera celeste desde el exterior, situación normal en la literatura científica. En estas circunstancias, es posible pensar en construir un modelo para los estudiantes de secundaria que haga viable comparar ambos puntos de vista y que también permita "hacer visibles" las líneas del cielo y una mejor comprensión del propio horizonte.

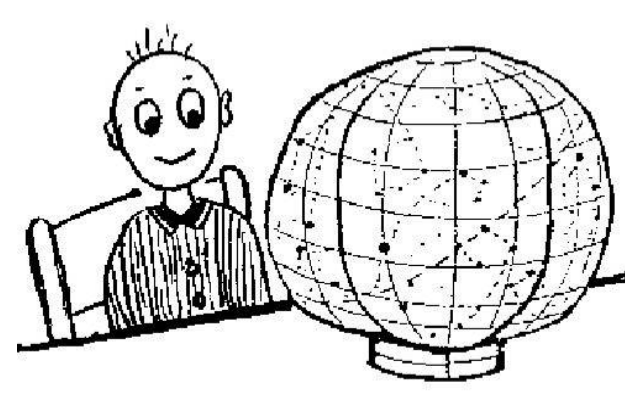

Figura 2: La esfera celeste desde el exterior.

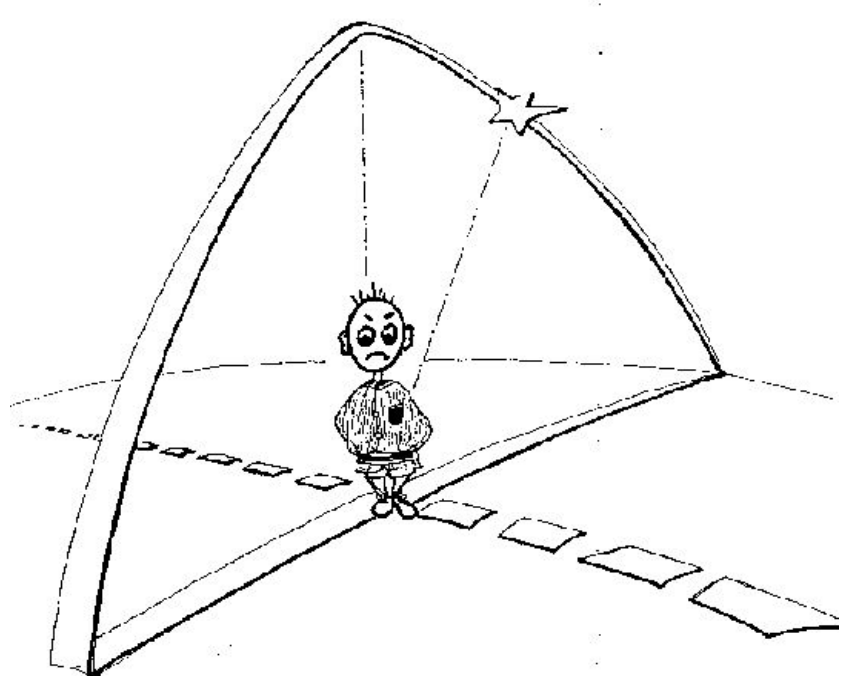

Figura 3: La esfera celeste desde el interior 


\section{Objetivos}

- Comprender el movimiento diurno y movimiento ánuo del Sol.

- Comprender el movimiento de la bóveda celeste.

- Comprender la construcción de un reloj de Sol elemental.

\section{Modelo local del horizonte}

Se empieza fotografiando el horizonte. Con una cámara sobre un trípode es muy sencillo tomar un conjunto de fotografías del horizonte desde algún lugar del patio del colegio -si las edificaciones colindantes lo permiten - o desde una terraza con el horizonte más despejado. (Señalaremos la posición del trípode marcándola sobre el suelo con pintura, para poder disponerlo de nuevo de forma exactamente igual). Es muy importante seleccionar muy bien el lugar porque la idea es situar allí el modelo durante cada observación. Al tomar cada fotografía es necesario que el encuadre tenga una zona común en la siguiente fotografía, para disponer después las copias sobre papel una sobre la otra y obtener el horizonte como una cadena de fotografías con continuidad.

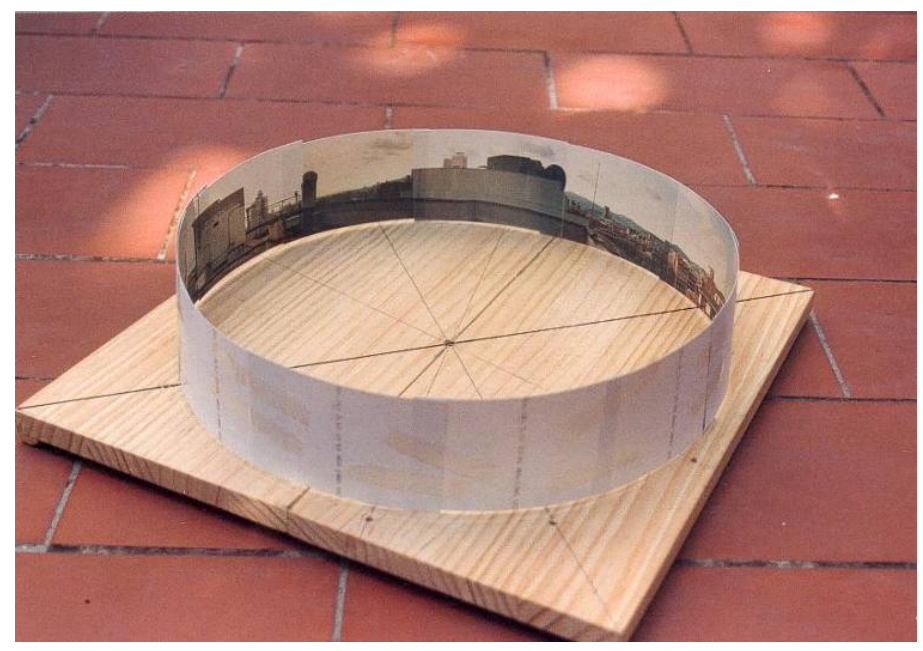

Figura 4: Modelo del horizonte de la escuela

Cuando tengamos las fotografías reveladas podemos fijar las copias una a continuación de la otra, formando un cilindro que después asentaremos sobre una base cuadrada de madera en el mismo lugar donde se han realizado las fotografías (Figuras 4 y 5). Es muy importante situar todas las fotografías acordes con el horizonte real.

A continuación se introduce el eje de rotación terrestre. Dando el valor de la latitud del lugar $\mathrm{f}$ se puede introducir en la maqueta un alambre según esta inclinación (Figuras 5 y 6 ). 


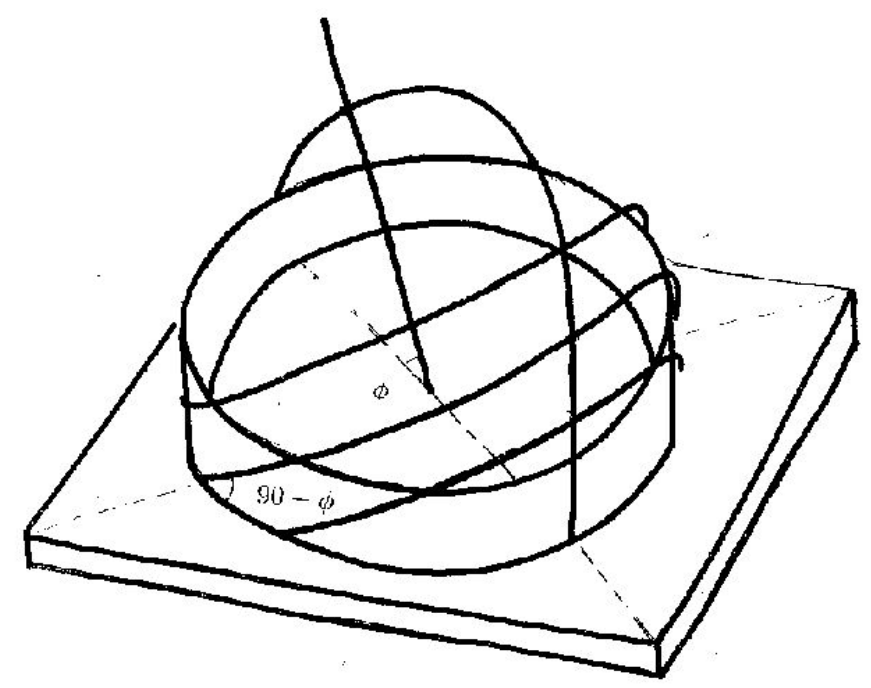

Figura 5: Modelo del horizonte de la escuela

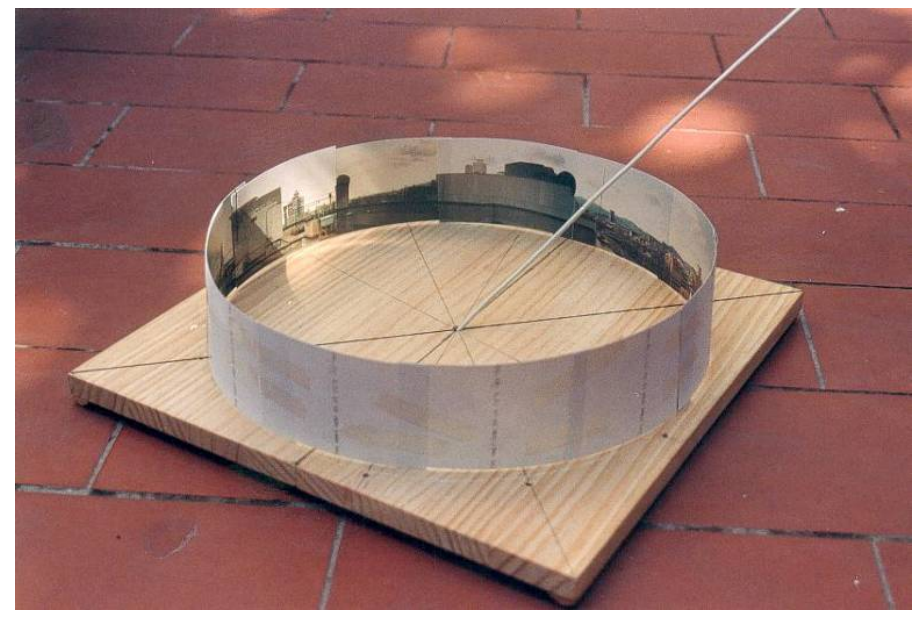

Figura 6: Eje de rotación terrestre

Con este dato es posible fijar el eje de rotación del modelo. Pero como el modelo está orientado según el horizonte local, la prolongación del alambre sirve para ayudar a visualizar el eje real y localizar el polo sur, y es útil también para imaginar la posición del punto cardinal sur. Obviamente introducir el punto cardinal Norte y el Polo Norte resulta fácil. A continuación se puede trazar la recta Norte-Sur sobre el modelo y también sobre el suelo del patio o la terraza donde se trabaja (usando el proceso normal de determinación de la recta Norte-Sur). Es muy importante porque cada vez que se vaya a usar el modelo habrá que orientarlo y es muy útil disponer de esta recta Norte-Sur real para facilitar el trabajo. (Con una brújula se puede comprobar dicha dirección). 


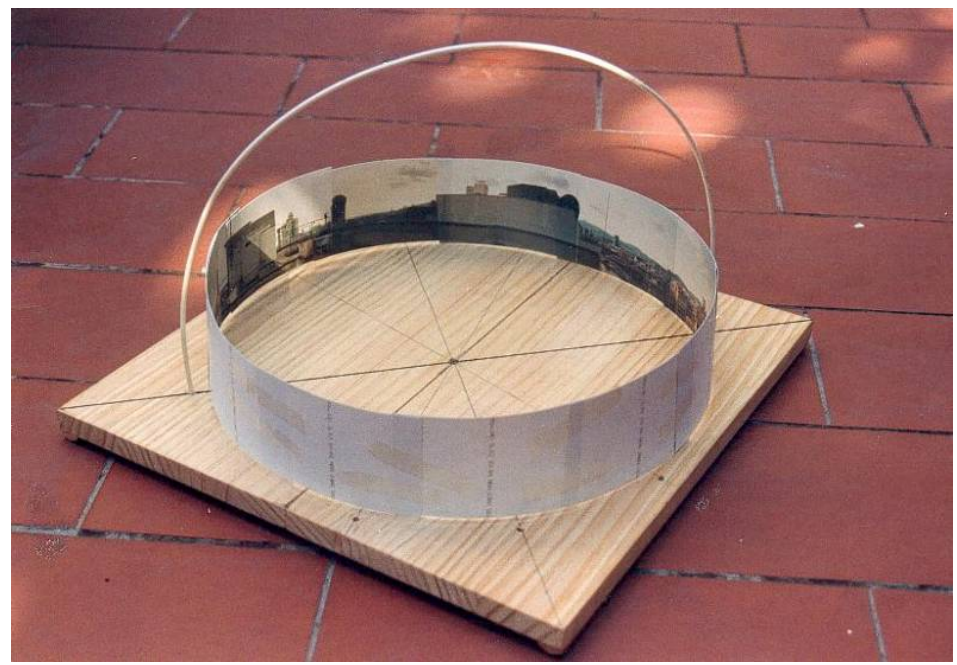

Figura 7: Meridiano del lugar

El siguiente objetivo consiste en situar el meridiano del lugar. El meridiano local es muy fácil de definir, pero no resulta un concepto simple de asimilar para los estudiantes (quizá, en parte, porque cada uno tiene su meridiano del lugar). Se puede fijar un alambre que pase por los puntos cardinales Norte y Sur y el eje de rotación de la Tierra (Figura 7). Este alambre es la visualización del meridiano del lugar en el modelo, pero permite imaginar sobre el cielo la línea del meridiano local. Ahora es muy fácil de imaginar porque empieza en los mismos lugares que el estudiante puede ver en el modelo. El meridiano local empieza en el mismo edificio que en la fotografía, pero en el horizonte real, y después de pasar por encima de su cabeza acabará en el mismo edificio que se visualiza gracias al alambre en el horizonte de fotografías.

Para introducir el ecuador el proceso es algo más complicado. Una posibilidad consiste en la línea Este-Oeste. Esta solución es muy sencilla, pero no aporta nada desde el punto de vista pedagógico. Para su aplicación a la enseñanza puede ser más conveniente usar de nuevo la fotografía. Se puede situar de nuevo la cámara sobre el trípode exactamente en la misma posición en que se tomaron las fotografías del horizonte en la primera ocasión en que se comenzó a desarrollar la maqueta. (Por este motivo se pinta en el suelo las marcas correspondientes para poder situar el trípode de nuevo en el mismo lugar). Con la cámara sobre el trípode se toma una foto de la salida y la puesta de Sol el primer día de primavera o de otoño. En este caso, tendremos dos instantáneas de la posición precisa de los puntos cardinales Este y Oeste respectivamente (Figura 8), respecto al horizonte de las fotografías y obviamente sobre el horizonte real.

El ecuador se simula por medio de un alambre perpendicular al eje de rotación terrestre que empieza y acaba en los puntos cardinales Este y Oeste (sobre el horizonte, en la recta perpendicular a la Norte-Sur). Pero no es sencillo fijar el círculo de alambre perpendicular al alambre que simboliza el eje de rotación, porque el eje de rotación está inclinado y obviamente el ecuador también deberá estarlo. Si se considera el Sol como una estrella más (el Sol es el más importante para los observadores porque está más próximo, pero su comportamiento no es diferente al de las otras estrellas) puede obtenerse la inclinación del movimiento de las estrellas cuando éstas se elevan o se ponen respecto al horizonte. Para ello basta con que se capten dos fotografías de este instante próximas al punto cardinal Este (Figuras 9 y 10) y al punto cardinal Oeste. 


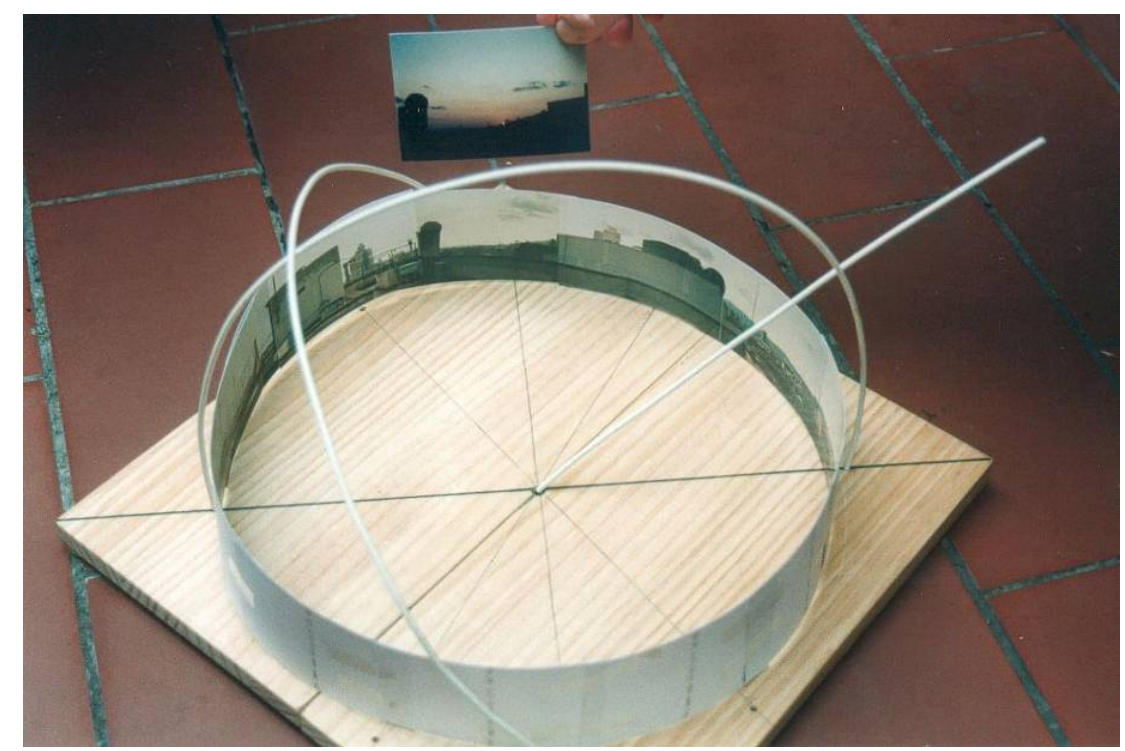

Figura 8: Linea del ecuador celeste

No es posible tomar las fotografías nocturnas mencionadas en el párrafo anterior desde la ciudad donde esta construida la escuela. Es preciso salir al campo, a un lugar suficientemente apartado sin contaminación luminosa. Hay que captar las fotografías con una cámara reflex, sobre un trípode y con un disparador de cable. Unos 10 minutos de tiempo de exposición son suficientes para la exposición nocturna (Figura 10). Para la salida del Sol es mejor usar el automático de la cama y dejar de disparar cuando moleste mirarlo (Figura 9) Es muy importante situar la cámara paralela al horizonte (se puede utilizar un nivel para realizar esta operación).

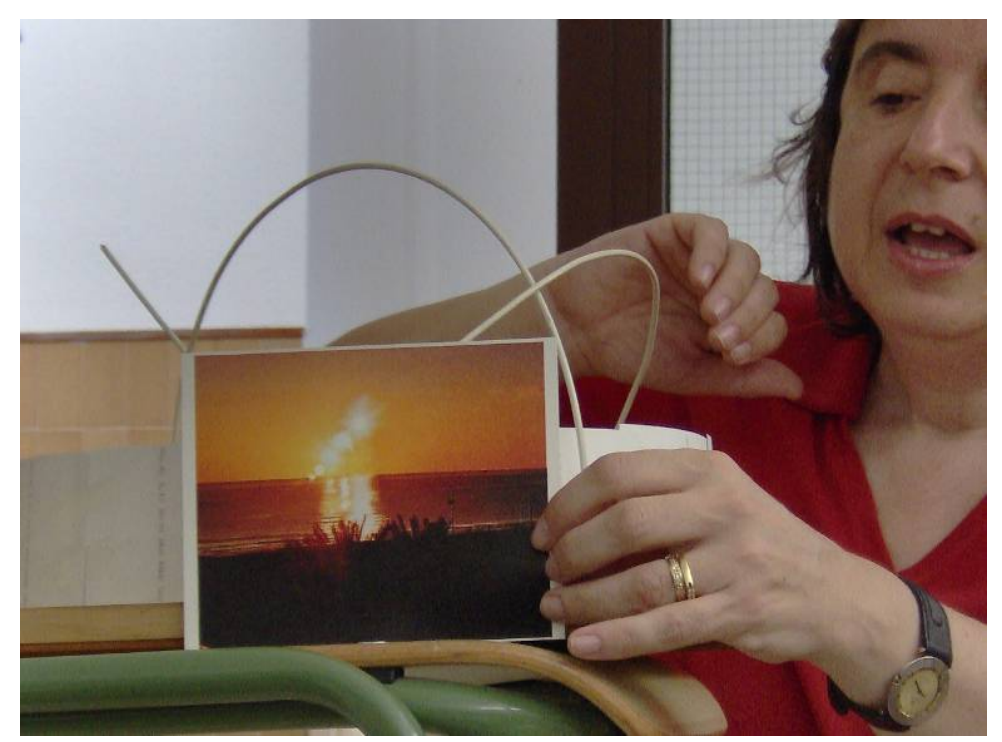

Figura 9: Fotografia del horizonte en el oeste 


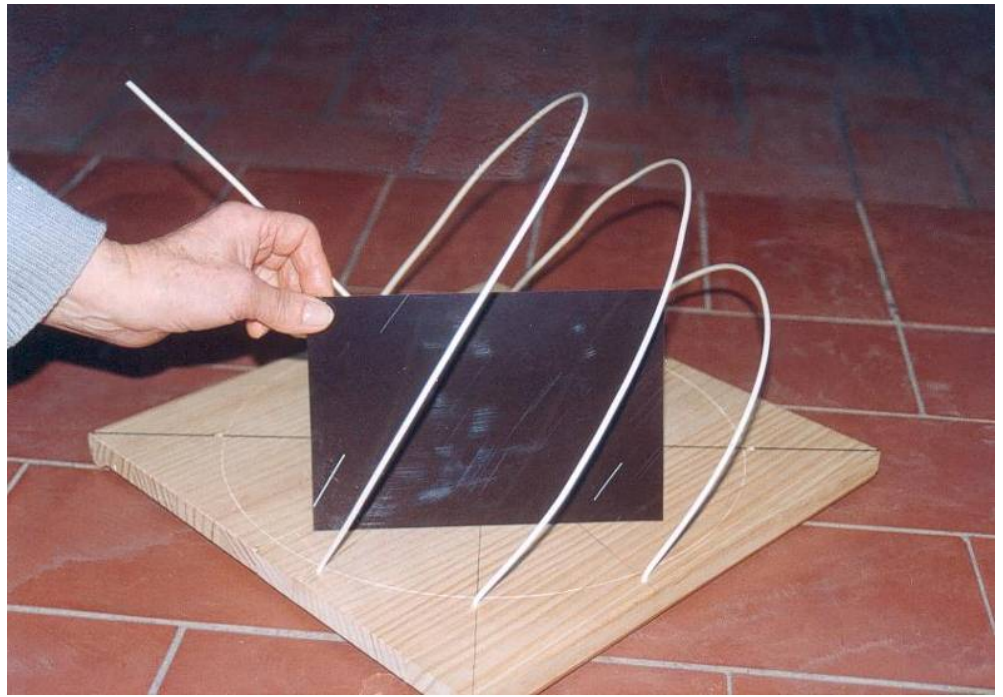

Figura 10: Fotografía nocturna del horizonte en el oeste

Es importante aprovechar esta ocasión para obtener un pequeño repertorio de fotografías. Por ejemplo se puede tomar una de la zona del polo dando unos 15 minutos de exposición, otra de la zona por encima de ella siguiendo el meridiano local, otra a continuación siguiendo también el mismo meridiano y así sucesivamente hasta conseguir la fotografía que ya está rasante al horizonte. La idea es fotografiar todo el meridiano local desde el polo Norte hasta el horizonte Sur pasando por encima de nuestras cabezas, si vivimos en el hemisferio norte. (Si vivimos en el hemisferio Sur, fotografiaremos el meridiano local desde el polo Sur hasta el horizonte Norte pasando por encima de nuestra posición). Evidentemente el meridiano local del lugar donde ha decidido tomar las fotografías no es el mismo que el de la escuela, pero los alumnos pueden comprender esta pequeña diferencia fácilmente.

Cuando se tienen todas las fotografías podemos construir una cinta del meridiano con todas ellas. Con esta cinta los estudiantes pueden comprender mejor el movimiento de la esfera celeste alrededor del eje de rotación de la Tierra. Es interesante ver que con el mismo tiempo de exposición la trayectoria dibujada por una estrella cambia de longitud. Es mínima en el entorno al polo y es máxima en el ecuador. También cambia de forma. En el ecuador la trayectoria dibuja una línea recta. En la zona próxima a la polar las líneas son curvas cóncavas y por debajo del ecuador son convexas. Si hacemos las copias sobre papel de las fotografías suficientemente grandes, podemos situar la cinta por encima de la cabeza del estudiante, lo que le permitirá visualizar y comprender mejor el movimiento.

Usando las dos fotografías de los puntos cardinales Este y Oeste es posible conocer la inclinación de las trazas de las estrellas en el ecuador, y por lo tanto es posible situar el alambre que simboliza el ecuador sin problemas. Se sabe los puntos donde fijarlo y también la inclinación, así es que puede sujetarse el alambre sobre la madera y también sujetarlo con el meridiano local (Figura 8).

Evidentemente es posible introducir la cinta de fotografías del meridiano local sobre el modelo. Es suficiente hacer algunas fotocopias y agujerearlas por el punto que indica la polar para poder introducir el eje de rotación. Se observa que el alambre del ecuador se corresponde con las trazas en línea recta que se tienen en la cinta (Figura $11)$. 


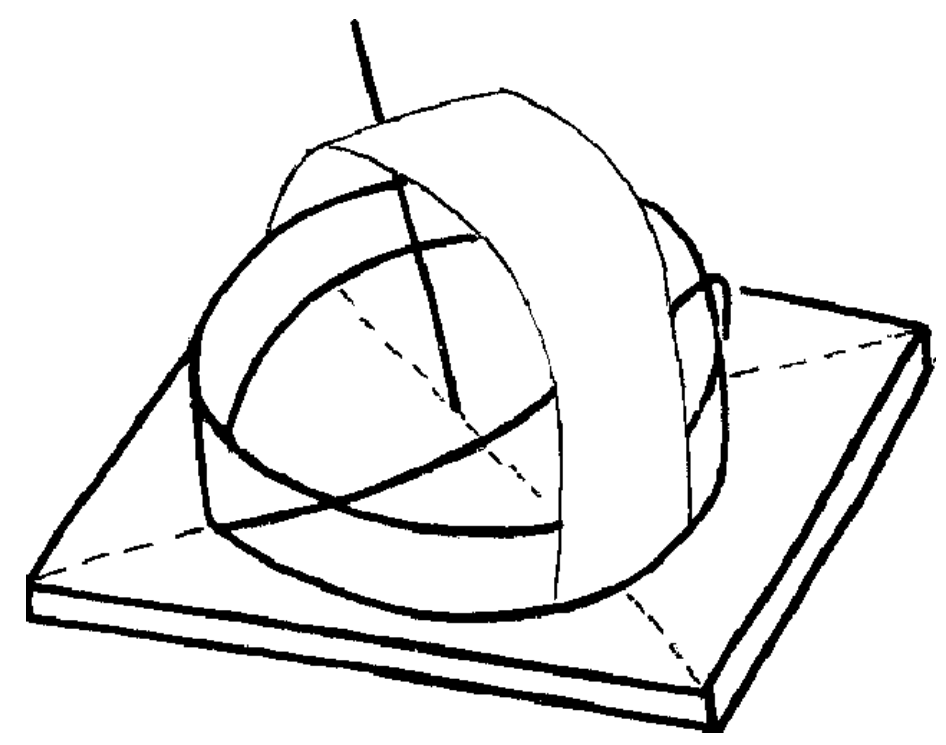

Figura 11: El meridiano local con fotografias

Con el modelo se puede ofrecer al estudiante las dos posibilidades de visualizar la esfera celeste desde el interior y desde el exterior.

Para la formación de los estudiantes es interesante que ellos puedan observar que el Sol no sale y se pone en la misma posición y que ésta no siempre coincide con el Este y el Oeste respectivamente. Hay muchos libros que mencionan que el Sol sale por el Este y se pone por el Oeste. Los estudiantes pueden ver que esto sólo es cierto dos veces al año, pero no lo es los días restantes (Figura 12).

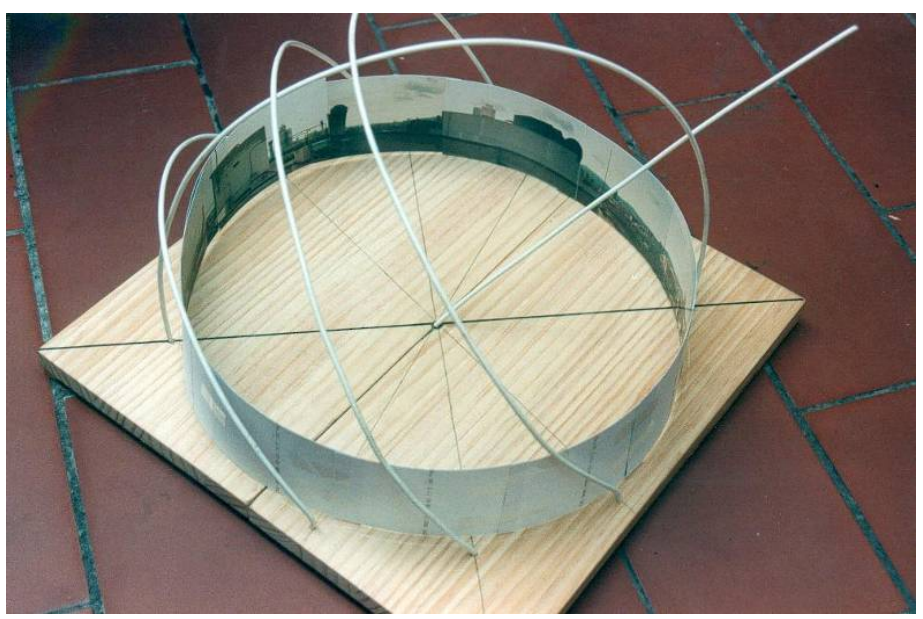

Figura 12: El meridiano local con fotografias

Si se toma de nuevo dos fotografías del primer día del invierno y del verano cuando el Sol sale y se pone, los alumnos podrán ver que las situaciones extremas en su ciudad son muy diferentes. Es sorprendente la diferencia que hay entre una y otra. También puede fijarse los paralelos de Cáncer y de Capricornio con las fotografías que proporciona la inclinación del ecuador, dado que los paralelos siguen esta misma inclinación. Con un simple transportador es posible verificar que el ángulo interior entre 
el paralelo de Cáncer y el ecuador es aproximadamente $23.5^{\circ}$, y que este ángulo es también el formado entre el ecuador y el paralelo de Capricornio (Figura 13).

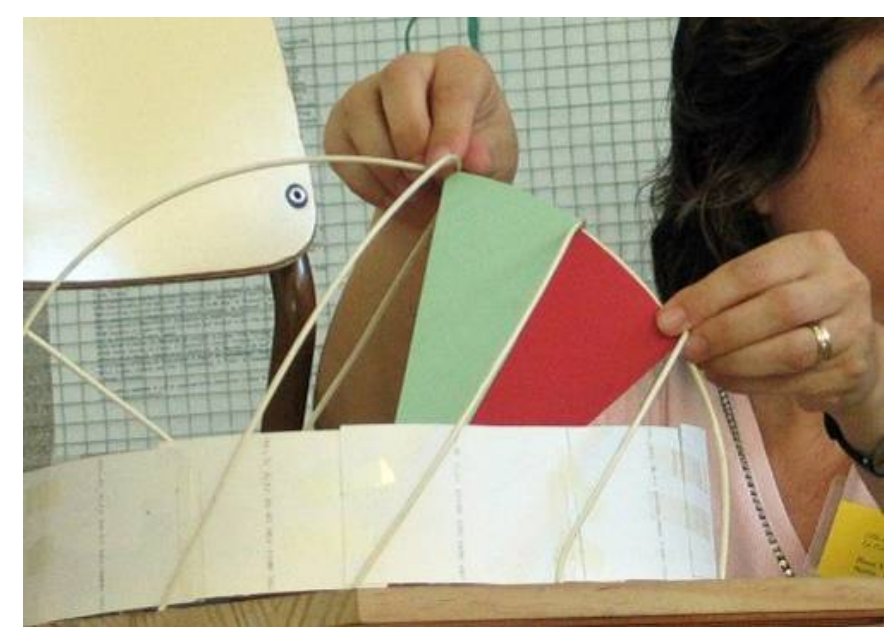

Figura 13: Los paralelos de Cáncer y Capricornio forman $23.5^{\circ}$ con el ecuador

De esta forma los alumnos ven de forma práctica y simultánea, la esfera desde el interior (la esfera real) y desde el exterior (el modelo). Con la ayuda de la maqueta los estudiantes pueden entender mejor su entorno, y las actividades de orientación realizadas desde la escuela se resuelven de forma muy sencilla. También pueden visualizar la zona que corresponde al movimiento del Sol, entre los paralelos de la maqueta e imaginarla sobre el cielo y el horizonte real de la ciudad. La orientación se convierte en un juego de niños.

\section{Relojes de Sol}

Pero hay otras posibilidades de aplicación del modelo. La maqueta no es más que un reloj de Sol, un gran reloj de Sol. Es fantástico para explicar de una forma sencilla y didáctica la construcción de un reloj considerando sólo el horizonte y el movimiento del Sol. En primer lugar es muy fácil ver que el eje de rotación de la Tierra se convierte en el estilete del reloj.

Si introducimos un plano en la dirección del plano ecuatorial y movemos una linterna sobre el paralelo de Cáncer, se puede ver la sombra del estilete (el alambre que representa el eje de rotación terrestre) recorriendo el plano del cuadrante ecuatorial, pero cuando se transita con la linterna sobre el paralelo de Capricornio entonces la sombra aparece en la zona de debajo del plano, y es evidente que cuando la linterna se sitúa sobre el ecuador no se obtiene sombra. Así pues resulta sencillo comprobar que el reloj ecuatorial funciona en verano y primavera mostrando las horas sobre el plano del reloj, en invierno y otoño debajo del mismo, y que hay dos días al año en que no funciona: los días de ambos equinoccios.

Si se considera el plano ecuatorial, el horizontal y el vertical orientado (EsteOeste), se puede ver que la linterna señala la misma hora en los tres cuadrantes. Además, puede observarse cuando son las horas de la mañana y de la tarde para el mismo estilete (el eje de rotación terrestre). Obviamente es la misma hora en los tres 
relojes (Figura 14). Se comprueba fácilmente en que zona hay que dibujar las horas de la mañana y de la tarde en cada reloj. (Todos los profesores han recibido alguna vez las horas mal dibujadas en un reloj solar; usando este modelo esto ya no sucede).

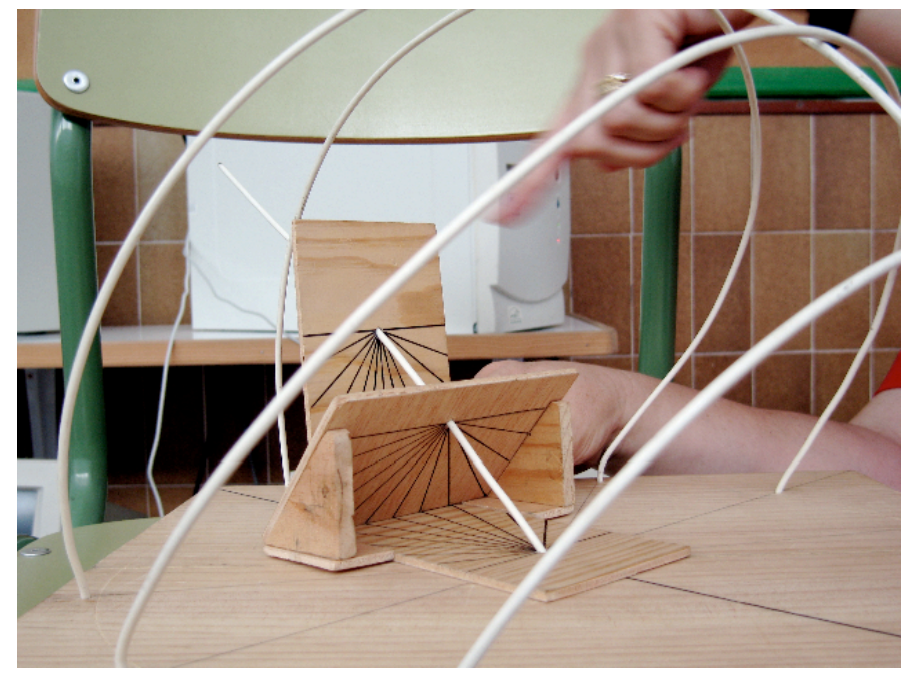

Figura 14: Los tres relojes de Sol

Al mover la linterna sobre los paralelos de Capricornio y Cáncer se ve fácilmente que el rayo de luz emitido por la lámpara produce sobre el plano una cónica diferente. En el primer caso (el primer día de verano) la cónica es casi una circunferencia y el área encerrada es claramente más pequeña que en el segundo caso. Cuando se sigue el otro paralelo (primer día de invierno) la sección es elíptica y el área encerrada es mucho mayor. Entonces los alumnos pueden comprender que la radiación está más concentrada en la primera situación, es decir que la temperatura superficial es mayor en verano, y como que también es evidente en el modelo que el número de horas de insolación solar es mayor, la consecuencia natural es que en verano hace más calor que en invierno (Figura 15).

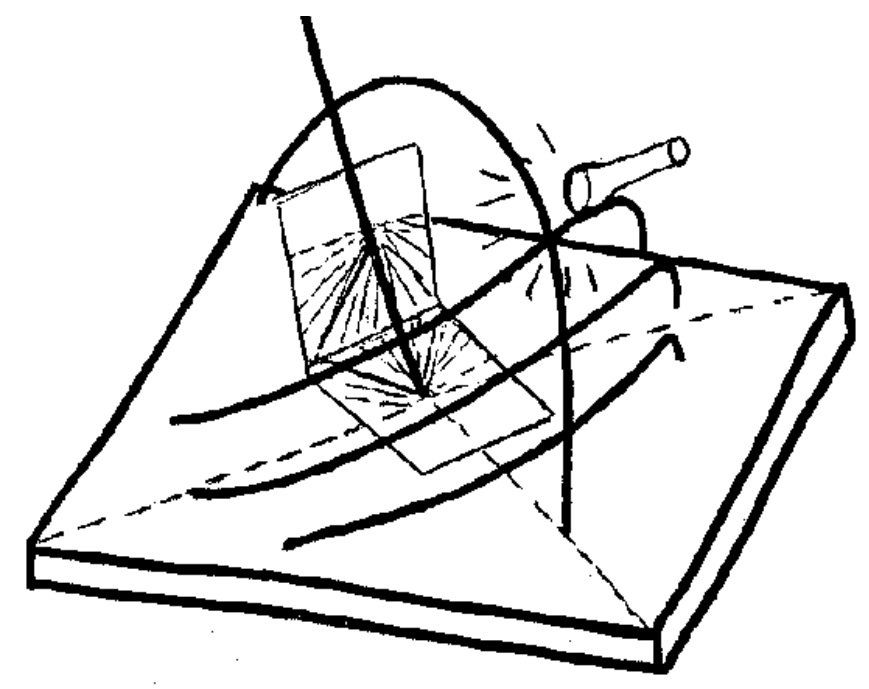

Figura 15: Los relojes y las estaciones 
Aprovecharemos esta oportunidad para mencionar algunos elementos que hay que conocer para poder construir un reloj de Sol.

El reloj ecuatorial es muy sencillo de realizar. Basta situar el estilete en la dirección del eje de rotación terrestre, esto es en la dirección Norte-Sur (una brújula nos puede ayudar a hacerlo) y con una altura sobre el plano del horizonte igual a la latitud del lugar (Figura 16). El estilete de cualquier reloj se situará siempre de la misma manera.

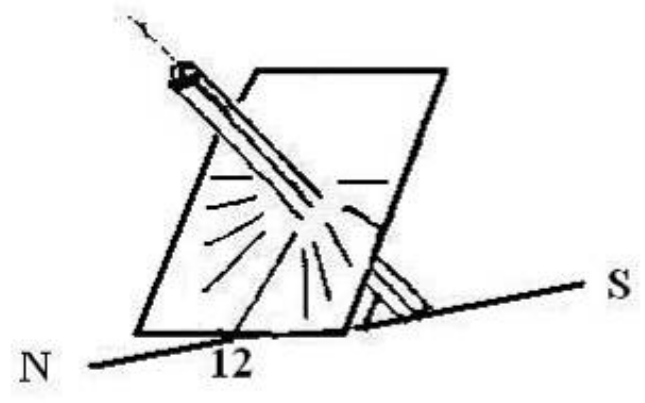

Figura 16: Reloj ecuatorial situado en estación (en el hemisferio norte).

Las líneas horarias del reloj ecuatorial se dibujarán a 15 grados (Figuras 17 y 18), ya que el Sol da una vuelta de $360^{\circ}$ en 24 horas. Si dividimos $360 / 24=15^{\circ}$ cada hora.

Las líneas horarias de un reloj horizontal o de un vertical orientado se obtienen por proyección del ecuatorial, sin mas que considerar la latitud del lugar (Figuras $17 \mathrm{y}$ 18)

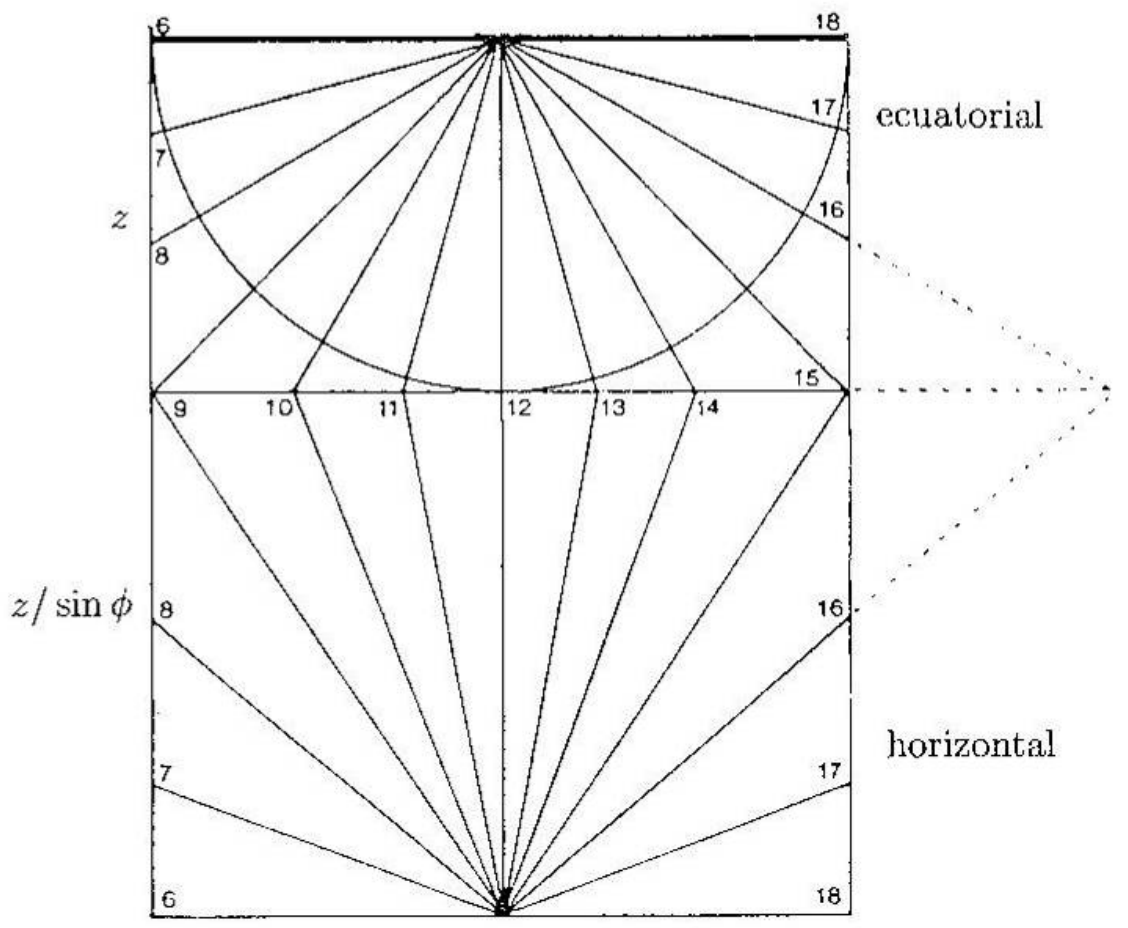

Figura 17: Relación existente entre el reloj ecuatorial y el horizontal (para $\phi=40^{\circ}$ ). 


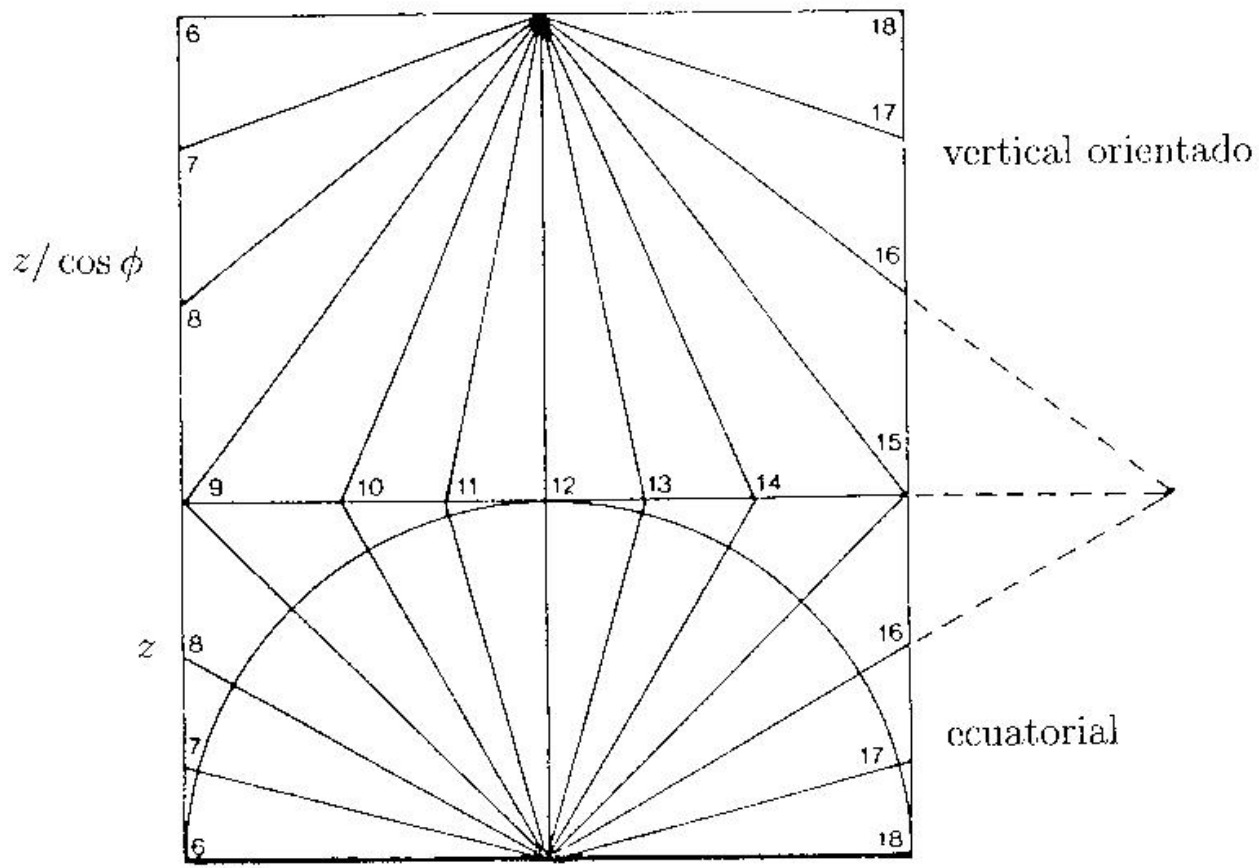

Figura 18: Relación existente entre el reloj ecuatorial y el vertical orientado (para $\phi=40^{\circ}$ ).

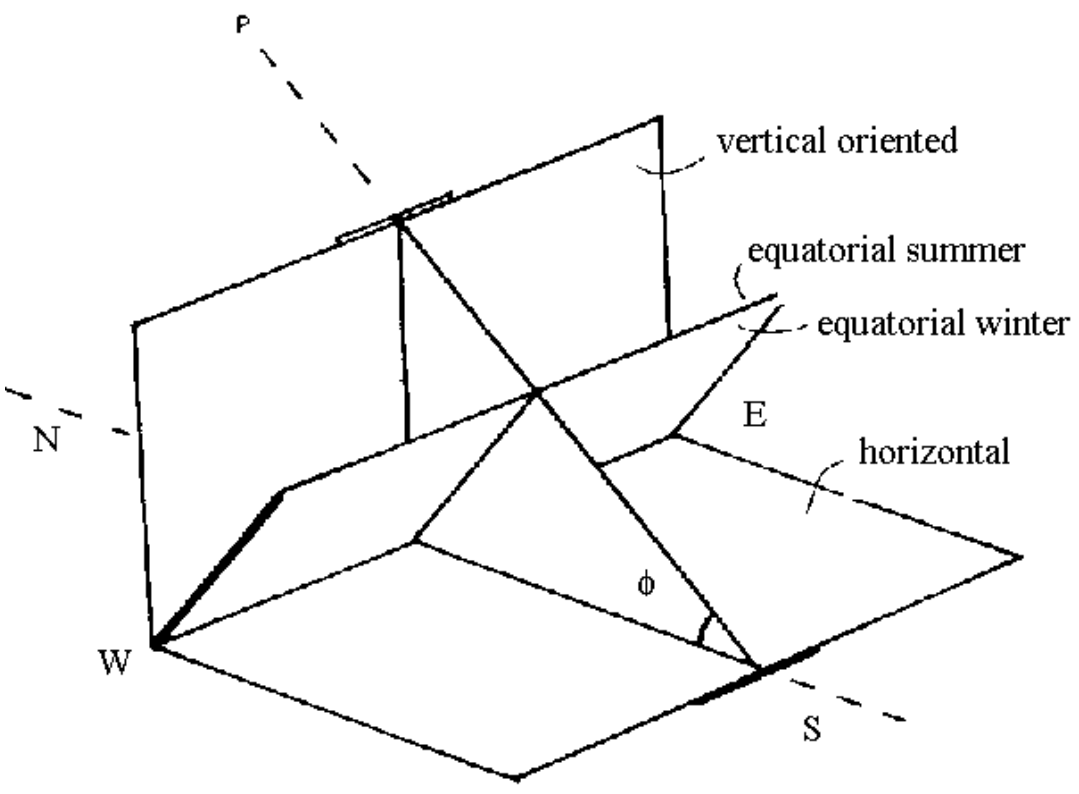

Figura 19: Sistema de tres relojes: ecuatorial, horizontal y vertical orientado.

Para construir el conjunto de relojes de la Figura 19 basta recortar las Figuras 20 y 21 por la línea exterior, doblar las diferentes zonas por las líneas horizontales y plegar el conjunto según el resultado que se puede ver en la Figura 19. 


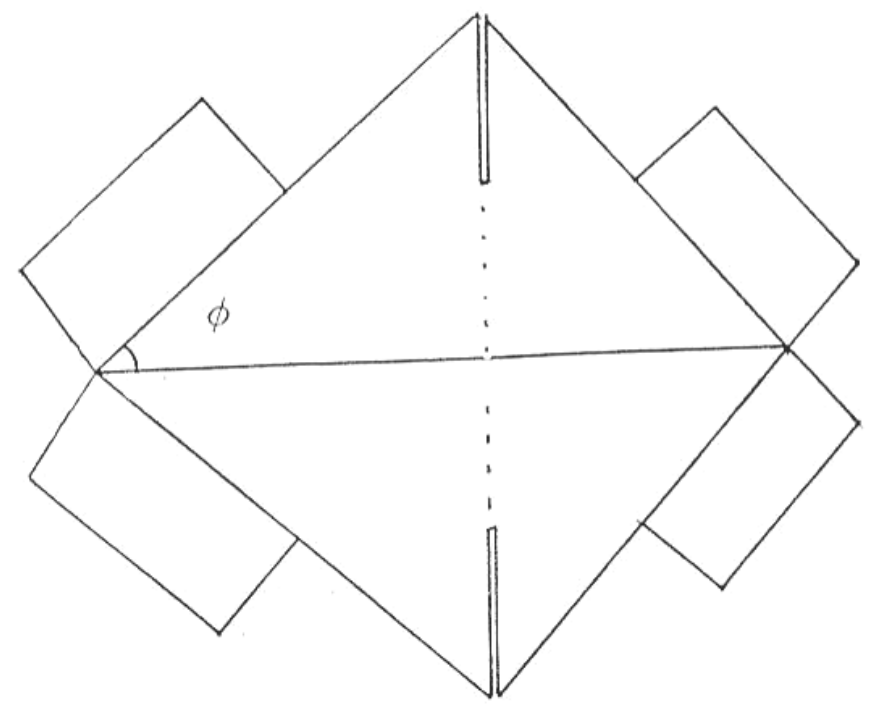

Figura 20: Triángulo de soporte del estilete del sistema de los tres relojes para latitud $\phi=40^{\circ}$.

\section{Tiempo solar y tiempo del reloj de "pulsera"}

Los relojes de Sol dan el tiempo solar, que no es el mismo que figura en los relojes que todos usamos en nuestra muñeca. Hay que considerar varios ajustes.

\section{Ajuste de Longitud}

El mundo se divide en 24 zonas de tiempo a partir del primer meridiano o meridiano de Greenwich. Para hacer el ajuste de longitud hay que conocer la longitud local y la longitud del meridiano estándar de su zona. Se añade con signo + hacia el Este y con signo - hacia el Oeste. Hay que expresar las longitudes en horas minutos y segundos $(1$ grado $=4$ minutos de tiempo).

\section{Ajuste de verano/invierno.}

Casi todos los países tienen el tiempo de verano y el de invierno. Se suele añadir una hora en verano. El cambio de horario de verano/invierno es una decisión del gobierno del país.

\section{Ajuste de la Ecuación de Tiempo}

La Tierra gira en torno al Sol según la ley de las áreas, es decir no es un movimiento constante, lo cual significa un serio problema para los relojes mecánicos. Así pues se define el tiempo medio (de los relojes mecánicos) como el promedio a lo largo de un año completo del tiempo. La Ecuación de Tiempo es la diferencia entre el «Tiempo Solar Real» y el «Tiempo Medio». Esta ecuación aparece tabulada en la Tabla 1.

\section{CONVERSIÓN DE TIEMPO}

Tiempo Solar + Ajuste Total $=$ Tiempo del reloj de pulsera 


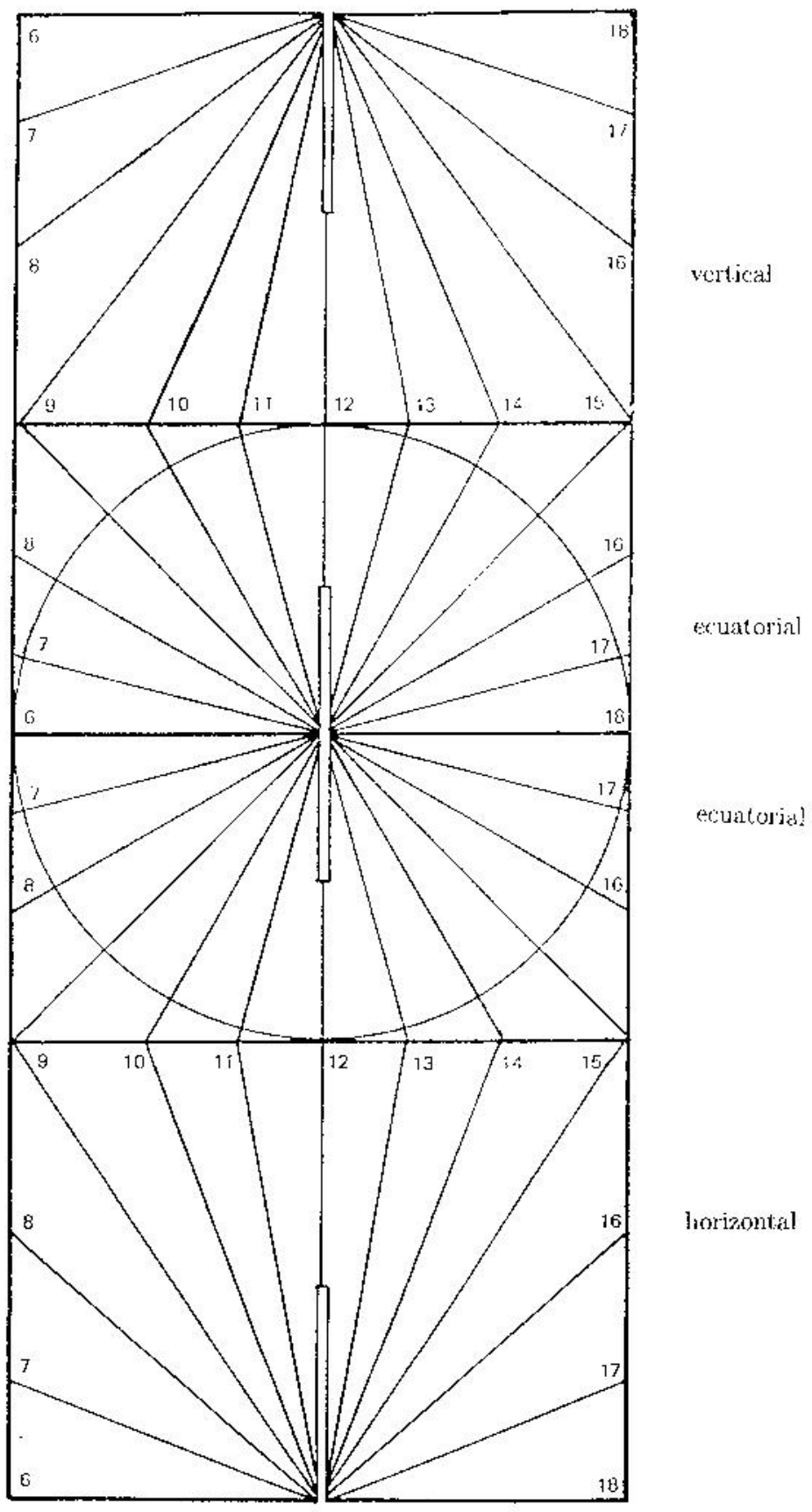

Figura 21: Plano del sistema de los tres relojes recortable (para $40^{\circ}$ de latitud). 

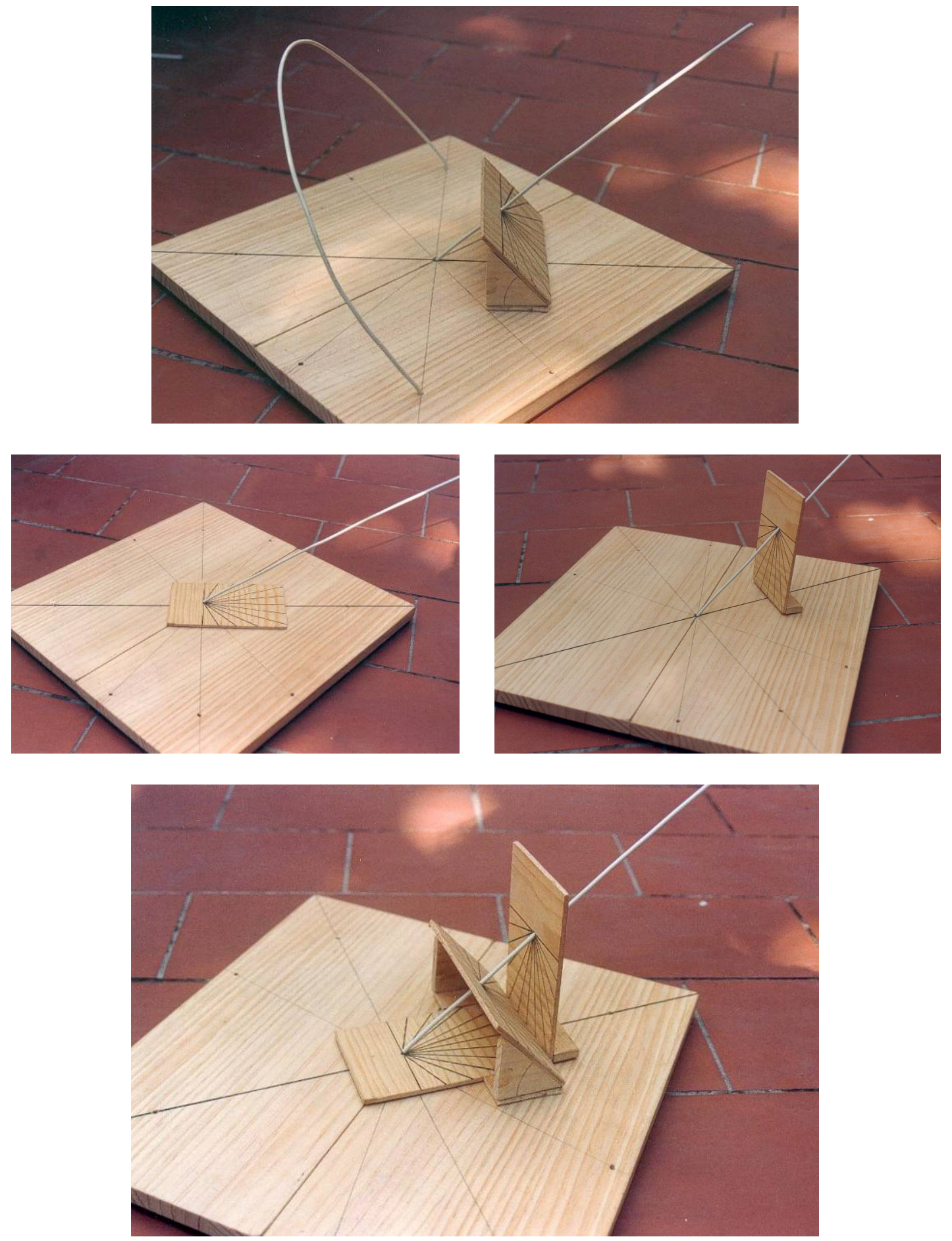

Figura 22: Los tres relojes construidos sobre la maqueta 
Tabla 1: Ecuacíon de Tiempo

\begin{tabular}{|c|c|c|c|c|c|c|c|c|c|c|c|c|}
\hline días & Ene. & Feb. & Mar. & Abr. & May & Jun. & Jul. & Ago. & Sep. & Oct. & Nov. & Dic. \\
\hline 1 & +3.4 & +13.6 & +12.5 & +4.1 & -2.9 & -2.4 & +3.6 & +6.3 & +0.2 & -10.1 & -16.4 & -11.2 \\
\hline 6 & +5.7 & +5.1 & +11.2 & +2.6 & -3.4 & -1.6 & +4.5 & +5.9 & -1.5 & -11.7 & -16.4 & -9.2 \\
\hline 11 & +7.8 & +7.3 & +10.2 & +1.2 & -3.7 & -0.6 & +5.3 & +5.2 & -3.2 & -13.1 & -16.0 & -7.0 \\
\hline 16 & +9.7 & +9.2 & +8.9 & -0.1 & -3.8 & +0.4 & +5.9 & +4.3 & -4.9 & -14.3 & -15.3 & -4.6 \\
\hline 21 & +11.2 & +13.8 & +7.4 & -1.2 & -3.6 & +1.5 & +6.3 & +3.2 & -6.7 & -15.3 & -14.3 & -2.2 \\
\hline 26 & +12.5 & +13.1 & +5.9 & -2.2 & -3.2 & +2.6 & +6.4 & +1.9 & -8.5 & -15.9 & -12.9 & +0.3 \\
\hline 31 & +13.4 & & +4.4 & & -2.5 & & +6.3 & +0.5 & & -16.3 & & +2.8 \\
\hline
\end{tabular}

Ejemplo 1: Barcelona (España) el 24 de Mayo.

\begin{tabular}{|c|l|c|}
\hline Ajuste & \multicolumn{1}{|c|}{ Comentario } & Resultado \\
\hline 1.Longitud & $\begin{array}{l}\text { Barcelona está en la misma zona estándar } \\
\text { que Greenwich. } \\
\text { Su longitud es } 2^{\circ} 10^{\prime} \mathrm{E}=2.17^{\circ} \mathrm{E}=-8.7 \mathrm{~m} \\
\left(1^{\circ} \text { es equivalente a } 4 \mathrm{~m}\right)\end{array}$ & $-8.7 \mathrm{~m}$ \\
\hline 2. Horario de verano & Mayo tiene horario de verano $+1 \mathrm{~h}$ & $+60 \mathrm{~m}$ \\
\hline 3. Ecuación de Tiempo & Leemos la tabla para el 24 de Mayo & $-3.6 \mathrm{~m}$ \\
\hline Total & & $+47.7 \mathrm{~m}$ \\
\hline
\end{tabular}

Por ejemplo a las $12 \mathrm{~h}$ de tiempo solar, nuestros relojes de "pulsera" señalan (Tiempo solar) $12 \mathrm{~h}+47.7 \mathrm{~m}=12 \mathrm{~h} 47.7 \mathrm{~m}$ (Tiempo del reloj de pulsera)

Ejemplo 2: Tulsa, Oklahoma (Estados Unidos) el 16 de Noviembre.

\begin{tabular}{|c|l|c|}
\hline Ajuste & \multicolumn{1}{|c|}{ Comentario } & Resultado \\
\hline 1.Longitud & $\begin{array}{l}\text { El meridiano estándar de Tulsa está a } 90^{\circ} \mathrm{W} . \\
\text { Su longitud es 95 } 58^{\circ} \mathrm{W}=96^{\circ} \mathrm{W} \text {, entonces } \\
\text { está a } 6^{\circ} \mathrm{W} \text { desde el meridiano estándar } \\
\left(1^{\circ} \text { es equivalente a } 4 \mathrm{~m}\right)\end{array}$ & $+24 \mathrm{~m}$ \\
\hline 2. Horario de verano & Noviembre no tiene horario de verano & \\
\hline 3. Ecuación de Tiempo & Leemos la tabla para el 16 de Noviembre & $-15.3 \mathrm{~m}$ \\
\hline Total & & $+8.7 \mathrm{~m}$ \\
\hline
\end{tabular}

Por ejemplo a las $12 \mathrm{~h}$ de tiempo solar, nuestros relojes de "pulsera"señalan (Tiempo solar) $12 \mathrm{~h}+8.7 \mathrm{~m}=12 \mathrm{~h} 8.7 \mathrm{~m}$ (Tiempo del reloj de pulsera) 


\section{La orientación}

Otro problema que se puede observar en los alumnos es el vinculado a sus dificultades de orientación. En un curso de astronomía general hay que conseguir instruirlos en el sentido de la orientación. Es posible que nuestros alumnos jamás estudien nuevamente astronomía. El mínimo resultado que hay que esperar de un curso (único) de astronomía consiste en que los alumnos sean capaces de reconocer dónde está el Norte, saber que la trayectoria del Sol está sobre el horizonte sur y que los planetas se mueven sobre este horizonte, y en particular que puedan ubicar los diferentes accidentes geográficos de su ciudad. Por ejemplo, sobre el horizonte de Barcelona (Figuras 23 y 24) los estudiantes pueden considerar diversas posibilidades relativas a la posición del Sol, la Luna y ciertas constelaciones sobre el horizonte. Las dos montañas que nosotros vemos están aproximadamente en posición opuesta. Pero para los estudiantes esto no significa nada y normalmente les cuesta distinguir que ciertos dibujos son posibles mientras que otros no se pueden dar jamás. Ellos conocen la teoría, pero la práctica no es suficiente si ellos no entienden las diferentes posibilidades.

La utilización del modelo pensado para resolver los inconvenientes mencionados en el apartado anterior resultó muy eficiente para aclarar muchas cuestiones relativas a la orientación en el horizonte local de una forma que inicialmente no estaba prevista.

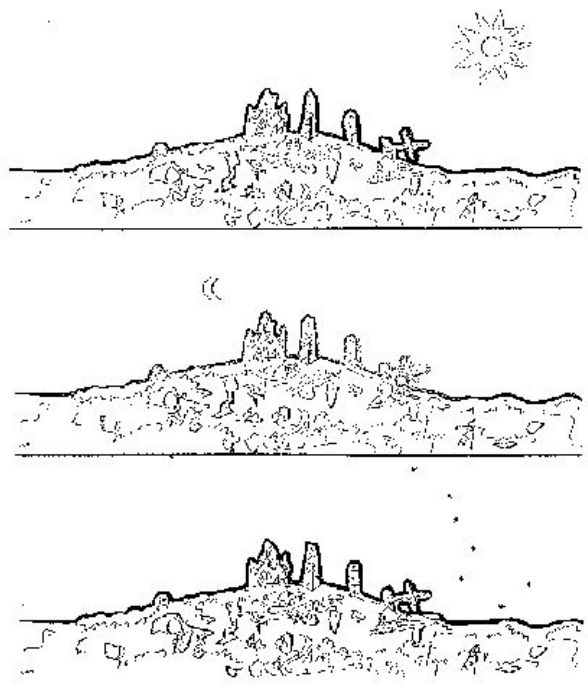

Figura 23 Horizonte Noroeste de Barcelona.

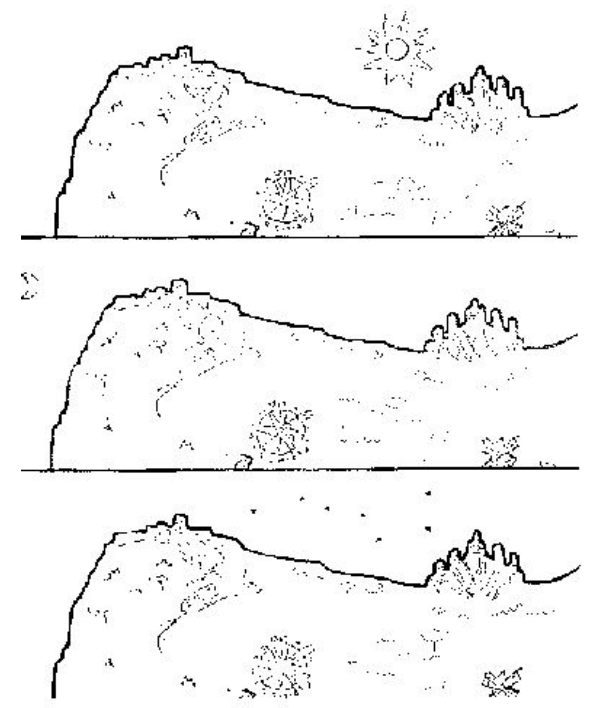

Figura 24 Horizonte Sudoeste de Barcelona.

Es bueno mencionar que este modelo es útil para explicar la situación local de la esfera celeste durante el día y durante la noche. Realmente sirve para comprender mejor el movimiento del Sol (y de otros miembros del Sistema Solar que se mueven en la zona próxima). Usando el modelo propuesto, los alumnos entienden que un astro brillante en la zona de la Polar nunca puede ser un planeta. 


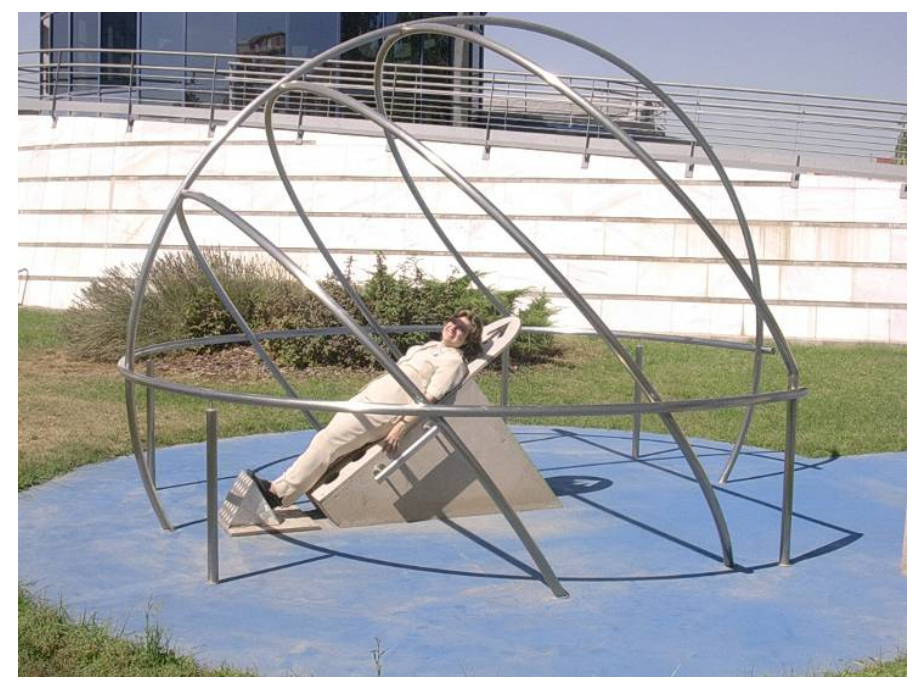

Figura 25: El modelo a gran escala en el Parque de las Ciencias de Granada (España).

Es una buena inversión producir un modelo como el explicado a gran escala. En ese caso los alumnos, e incluso los adultos pueden meterse dentro y verificar la posición del Sol en comparación con el ecuador y los paralelos que corresponden al primer día de los solsticios de verano e invierno. En algunos museos de la ciencia ya se han construido este tipo de modelo (Figura 25). Después de usar el modelo los alumnos pueden discernir contenidos que antes no se les habrían ocurrido. Les queda, por ejemplo, muy claro que el Sol no sale y se pone perpendicularmente al horizonte salvo en el ecuador.

\section{Bibliografía}

LANCIANO, N., Sopra l'orizzonte. Materiali per l'insegnamento dell'Astronomia nella scuola dell'obligo, Laboratorio di Didattica delle Scienze, Universita di Roma "La Sapienza", Roma, 1989.

LANCIANO, N., Dentro il cielo. Materiali per l'insegnamento e per l'aggiornamento degli insegnanti in Astronomia. Laboratorio di Didattica delle Scienze, Universita di Roma "La Sapienza", Roma, 1992.

ROS, R.M., De l'intérieur et de l'extérieur, Les Cahiers Clairaut, 95, p.1-5, Orsay, 2001 .

ROS, R.M., Laboratorio de Astronomía, Tribuna de Astronomía, 154, p.18-29, Madrid, 1998.

ROS, R.M., Sunrise and sunset positions change every day, Proceedings of 6th EAAE International Summer School, 177, 188, Barcelona, 2002.

ROS, R.M., CAPELL, A., COLOM, J., "El planisferio y 40 actividades más". Barcelona: Antares, 2005. 
Rosa M. Ros

ROS, R.M., LANCIANO, N., El horizonte en la Astronomía, Astronomía Astrofotografía y Astronáutica, 76, p.12-20, Madrid, 1995. 